\title{
Wedge-shaped microfluidic chip for circulating tumor cells isolation and its clinical significance in gastric cancer
}

\author{
Chaogang Yang ${ }^{1,2,3+}$, Nangang Zhang ${ }^{4 \dagger}$, Shuyi Wang ${ }^{1,2,3}$, Dongdong Shi ${ }^{1,2,3}$, Chunxiao Zhang ${ }^{1,2,3}$, Kan Lii ${ }^{4,5^{*}}$ \\ and Bin Xiong ${ }^{1,2,3^{*}}$
}

\begin{abstract}
Background: Circulating tumor cells (CTCS) have great potential in both basic research and clinical application for the managements of cancer. However, the complicated fabrication processes and expensive materials of the existing CTCs isolation devices, to a large extent, limit their clinical translation and CTCs'clinical value. Therefore, it remains to be urgently needed to develop a new platform for achieving CTCs detection with low-cost, mass-producible but high performance.

Methods: In the present study, we introduced a novel wedge-shaped microfluidic chip (named CTC- $\triangle$ Chip) fabricated by two pieces of glass through wet etching and thermal bonding technique for CTCs isolation, which achieved CTCs enrichment by different size without cell surface expression markers and CTCs identification with three-color immunocytochemistry method (CK+/CD45-/Nucleus+). We validated the feasibility of CTC- $\triangle$ Chip for detecting CTCs from different types of solid tumor. Furthermore, we applied the newly-developed platform to investigate the clinical significance of CTCs in gastric cancer (GC).

Results: Based on "label-free" characteristic, the capture efficiency of CTC- $\triangle$ Chip can be as high as $93.7 \pm 3.2 \%$ in DMEM and $91.0 \pm 3.0 \%$ in whole blood sample under optimized conditions. Clinically, CTC- $\Delta$ Chip exhibited the feasibility of detecting CTCs from different types of solid tumor, and it identified 7.30 7.29 CTCs from $2 \mathrm{~mL}$ peripheral blood with a positive rate of $75 \%$ (30/40) in GC patients. Interestingly, we found that GC CTCs count was significantly correlated with multiple systemic inflammation indexes, including the lymphocyte count, platelet count, the level of neutrophil to lymphocyte ratio and platelet to lymphocyte ratio. In addition, we also found that both the positivity rate and CTCs count were significantly associated with multiple clinicopathology parameters.

Conclusions: Our novel CTC- $\triangle$ Chip shows high performance for detecting CTCs from less volume of blood samples of cancer patients and important clinical significance in GC. Owing to the advantages of low-cost and mass-producible, CTC- $\triangle$ Chip holds great potential of clinical application for cancer therapeutic guidance and prognostic monitoring in the future.
\end{abstract}

Keywords: Circulating tumor cells, Cell capture, Wedge-shaped chip, Microfluidic, Gastric cancer

\footnotetext{
*Correspondence: liukan2002@gmail.com; binxiong1961@whu.edu.cn

${ }^{\dagger}$ Chaogang Yang and Nangang Zhang contributed equally to this work

${ }^{1}$ Department of Gastrointestinal Surgery \& Department of Gastric

and Colorectal Surgical Oncology, Zhongnan Hospital of Wuhan

University, No.169 Donghu Road, Wuchang District, Wuhan 430071, China

${ }^{4}$ College of Electronic and Electrical Engineering, Wuhan Textile

University, No.1 Sunshine Avenue, Hongshan District, Wuhan 430200,

China

Full list of author information is available at the end of the article
}

(c) The Author(s) 2018. This article is distributed under the terms of the Creative Commons Attribution 4.0 International License (http://creativecommons.org/licenses/by/4.0/), which permits unrestricted use, distribution, and reproduction in any medium, provided you give appropriate credit to the original author(s) and the source, provide a link to the Creative Commons license, and indicate if changes were made. The Creative Commons Public Domain Dedication waiver (http://creativecommons.org/ publicdomain/zero/1.0/) applies to the data made available in this article, unless otherwise stated. 


\section{Background}

Circulating tumor cells (CTCs) are cancer cells that break away from the primary site and disseminate into blood stream. These "break away" cells, circulate and survive in the peripheral blood, potentially seed into distant organs and finally form the vital metastases-the main cause of cancer-related death. Recently, CTCs' detection has been considered as a "liquid biopsy", which could supply important information for clinical practice, such as treatment response monitor, potential metastasis prediction and prognosis evaluation in varieties of malignant tumors, including breast [1], prostate [2], gastric [3], colorectal [4] and lung cancer [5]. However, due to the extremely low concentration of CTCs (one in millions of blood cells) [6, 7], CTCs' detection has always been technically challenging.

Over the past decades, numerous separation technologies had been developed based on different biological and physical properties of CTCs [8-17]. The CellSearch ${ }^{\mathrm{TM}}$ system (Veridex, LLC, Raritan, NJ, USA), depending on epithelial cell adhesion molecule (EpCAM) marker to enrich and isolate CTCs [18], was the first and only semiautomated CTCs enumeration assay approved by FDA for the diagnosis of metastatic breast, prostate, and colorectal cancers. Nevertheless, EpCAM-based methods may loss parts of CTCs undergoing epithelial-to-mesenchymal transition (EMT) [19], which might not appropriate to get a more comprehensive spectrum of CTCs. In addition, because of the expensive antigen, label-based methods were limited for clinical use. Recently, isolation by size of epithelial tumor cells (ISET) technology had been widely used as a new set of tools for CTCs capture and achieved higher CTCs detection sensitivity $[10,20$, 21]. Based on this method, our ${ }^{\text {os } I S E T ~ d e v i c e ~[22] ~ s h o w e d ~}$ high efficiency of CTCs isolation in several types of solid

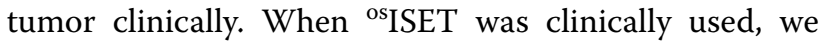
found that the size heterogeneity of CTCs leads to miss parts of CTCs (diameter $<8 \mu \mathrm{m}$ ). For gastric cancer (GC), although several studies demonstrated that CTCs' detection could be utilized for judging tumor stage, predicting patients' survival and monitoring therapeutic response [23-25], but the drawbacks of the above methods limited its further clinical application to validate the significant role of CTCs.

Herein, we fabricated a low-cost wedge-shaped microfluidic chip (named CTC- $\Delta$ Chip) to efficiently isolate CTCs from blood. Owning to the special structure of the microfluidic chamber, the size cut-off for enriching CTCs in the CTC- $\Delta$ Chip was able to be designed as small as $5 \mu \mathrm{m}$ [26] and CTCs even with slightly differential of biophysical properties could be entrapped and located at the microfluidic chamber with large space distribution. What's more, the CTC- $\Delta$ Chip still maintained a large loading volume of peripheral blood and quick sample processing. In this study, we demonstrated two aspects: (1) The feasibility and capture efficiency of CTC- $\Delta$ Chip for CTCs detection from the whole blood samples of different types of solid cancer patients; (2) The potential clinical value and significance of CTCs isolated by it in GC patients.

\section{Methods \\ Preparation of CTC- $\Delta$ Chip}

CTC- $\Delta$ Chip was fabricated by wet etching technique and thermal bonding technique. Details were showed in Additional file 1. As shown in Fig. 1a, CTC- $\Delta$ Chip was composed of a wedge-shaped microchamber, two inlets, a linear reservoir and one outlet. The wedgeshaped microchamber was the core functional part of the CTC- $\Delta$ Chip to isolate CTCs from patient's whole blood. The detailed structure parameters of CTC$\Delta$ Chip were shown in Fig. 1b, and the schematic diagram of CTC isolation using the CTC- $\Delta$ Chip was shown in Fig. 1c. To investigate how the outlet height (size cut-off for enrich CTCs) of microchamber affected the capture efficiency, CTC- $\Delta$ Chip with different outlet heights $(4,5,6,7,8 \mu \mathrm{m}$, respectively) were fabricated. Finally, CTC- $\Delta$ Chip was assembled into a small microfluidic device, the detailed structure of which was shown in Additional file 2.

\section{Cancer cell and human blood sample preparation}

BGC823 (human gastric cancer cell), HCT116 (human colorectal cancer cell), PC3 (human prostate cancer cell) and SKBR3 (human breast cancer cell) were obtained from Hubei Key Laboratory of Tumor Biological Behaviors. Cells were cultured in Dulbecco's modified eagle medium (DMEM, Hyclone, Thermo scientific, USA) added with $10 \%$ fetal bovine serum (Sigma, USA) and $1 \%$ penicillin/streptomycin at $37{ }^{\circ} \mathrm{C}$ in $5 \%$ humidified $\mathrm{CO}_{2}$ incubator. Adherent cells were released with $0.25 \%(\mathrm{w} / \mathrm{v})$ trypsin (Gibco, USA), resuspended in DMEM medium, assessed for concentration with a hemocytometer, and rocked gently on a shaker 5 min prior to experiments. Whole blood samples from healthy donors and patients were obtained from Department of Clinical Laboratory, Zhongnan Hospital of Wuhan University according to a protocol by the Institutional Review Board (IRB). All blood specimens were collected into the vacutainer tubes (BD, New Jersey, USA) containing EDTA-K2 and finished the experiments within $3 \mathrm{~h}$. All the participants have provided their written informed consent to participate in this study. This research was approved by the Medical Ethical Committee of Zhongnan Hospital. 


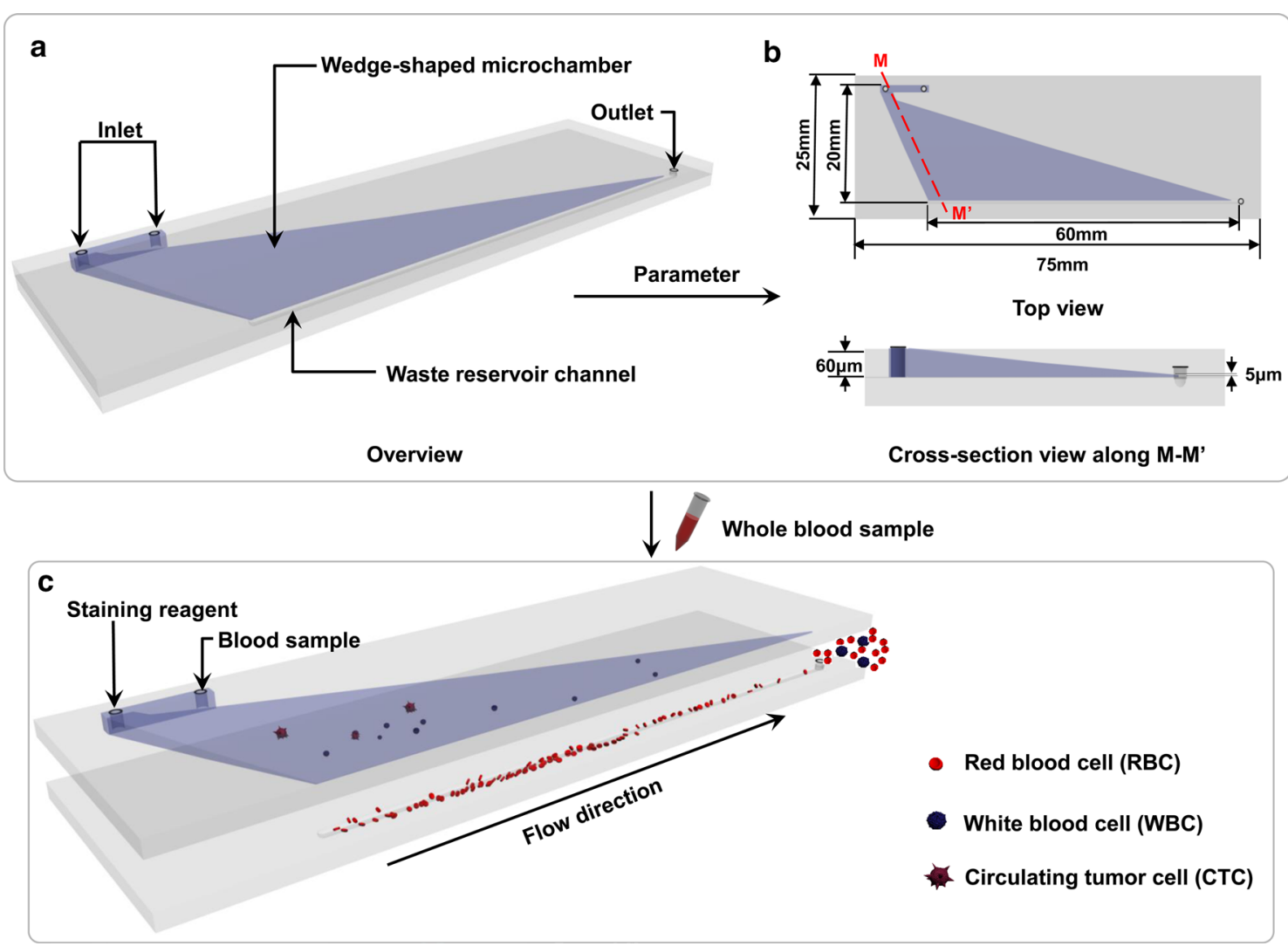

Fig. 1 Schematic diagram of CTCS isolation using the wedge-shaped microfluidic chip. a Overview of the wedge-shaped microfluidic chip; b detailed structural parameters of the microfluidic chip; c schematic diagram of CTCs isolation using the microfluidic chip with a wedge-shaped microchamber

\section{Optimization assays}

Spiked samples containing target cells (BGC823, 100 cells $2 \mathrm{~mL}^{-1}$ DMEM, pre-stained with CK) were injected into CTC- $\Delta$ Chip with different outlet heights $(4,5,6,7$, $8 \mu \mathrm{m}$, respectively) at flow rate of $200 \mu \mathrm{L} / \mathrm{min}$ to investigate the effect of outlet height on the capture efficiency. Finally, the spiked samples were injected into CTC- $\Delta$ Chip with $5 \mu \mathrm{m}$ outlet height at different flow rate $(50,100,200,300,400,600,800 \mu \mathrm{L} / \mathrm{min}$, respectively) to investigate the effect of flow rate on the capture efficiency.

\section{Sample processing and performance validation}

BGC823, HCT116, PC3 and SKBR3 cells were spiked into DMEM medium and blood sample at concentrations of 200 cells per $2 \mathrm{~mL}$. Just prior to cell capture, $4 \%$ paraformaldehyde (PFA) solution was added into whole blood samples in a 1:10 v/v ratio, and incubated for $10 \mathrm{~min}$ at room temperature. Then, the spiked samples were introduced into the CTC- $\Delta$ Chip by use of syringe pump in optimal cell-capture condition. Each test had been repeated for three times. After sample loading, CTC- $\Delta$ Chip was rinsed with PBS solution at $200 \mu \mathrm{L} /$ min for $10 \mathrm{~min}$. After rinsing, followed by staining of three-color immunocytochemistry method for CK, CD45 and nucleus, specifically captured cancer cells were identified and counted on the substrates. Finally, we imaged and enumerated targeted cells using a highcontent automatic screening system (CX7, CellInsight, ThermoFisher Scientific, USA).

\section{Cell capture from healthy and patient's blood samples}

Cell capture efficiency was validated using the CTC$\triangle$ Chip to capture target cells (BGC823) at concentrations of 50,100,150, 200 cells per $2 \mathrm{~mL}$ from the two kinds of sample: (1) $2 \mathrm{~mL}$ DMEM, and (2) $2 \mathrm{~mL}$ human blood (healthy donor). Patient blood samples $(2 \mathrm{~mL})$ were processed by the CTC- $\Delta$ Chip according to the procedure described above. Captured cells were rinsed with PBS and then stained by three-color immunocytochemistry method for CK, CD45 and nuclear staining. 


\section{Data collection}

Clinicopathologic data of patients were collected from hospital information system, including age, gender, tumor location, pathological type, differentiation degree, lymphovascular invasion, perineural invasion, tumor depth, lymph node status, metastasis status, TNM stage, and the level of Ki-67 and tumor markers. The clinicopathological classifications of cancers were determined according to the 7th edition of the American Joint Committee on Cancer/International Union Against Cancer tumor-node-metastasis (TNM) classification system [27].

\section{Statistical analysis}

Categorical data displayed in a contingency table were analyzed using Fisher's exact test. Continuous data were analyzed by Mann-Whitney test. Nonparametric correlation analysis used the method of Spearman rank correlation analysis. All statistical analyses were performed with the IBM SPSS 22.0 statistical software package (IBM Inc.). $\mathrm{P}<0.05$ was considered statistically significant.

\section{Results}

\section{Characterization and optimization of CTC- $\Delta$ Chip}

Figure 1 illustrated the schematic workflow of CTC$\Delta$ Chip to efficiently enrich the CTCs from whole blood samples based on the biophysical property. The wedgeshaped microchamber in CTC- $\Delta$ Chip was the core function part. The height of the microchamber in CTC- $\Delta$ Chip was designed to gradually decrease (Fig. 1b). The integrated process of CTCs isolation using the CTC- $\Delta$ Chip was shown in Additional file 3. To obtain high capturing efficiency of CTCs and ensure the minimum loading volume of blood sample $(2 \mathrm{~mL})$ at the same time, optimization studies were conducted to determine a reasonable outlet height (Fig. 2A). Blood samples were collected and stored in $4{ }^{\circ} \mathrm{C}$ for 6,24 and $48 \mathrm{~h}$ individually. The loading volume of patient blood sample was measured. When the cut-off size of CTC- $\Delta$ Chip was $5 \mu \mathrm{m}$, the minimum loading volume of blood sample was more than 10, 7.6 and $3.6 \mathrm{~mL}(\mathrm{n}=5)$. Although the capture efficiency of BGC823 cells in DMEM decreased from around 96.7$74 \%$ with outlet height increasing from 4 to $8 \mu \mathrm{m}$, it's still more than $93.7 \%$ in outlet height of $5 \mu \mathrm{m}$ (Fig. 2B). Considering of capture efficiency and minimum blood loading volume, the optimal cut-off size of the CTC- $\Delta$ Chip would be $5 \mu \mathrm{m}$. The relationship of capture efficiency and flow rate had been studied. As we known, the flow rate would decide the whole completion time of sample processing. Thus, we validated the cell capture efficiency of CTC- $\Delta$ Chip using optimized outlet height in different flow rates. As observed in Fig. 2C, the capture efficiencies decreased from 97.7 to $40.3 \%$ when the flow rates increased from 50 to $800 \mu \mathrm{L} / \mathrm{min}$. When the flow rate was $200 \mu \mathrm{L} / \mathrm{min}$, the capture efficiency was up to $93 \%$. In order to achieve the balance of completion time and capture efficiency, $200 \mu \mathrm{L} / \mathrm{min}$ was chosen for the optimal flow rate of the CTC- $\Delta$ Chip.

\section{Performance evaluation of CTC- $\Delta$ Chip}

Under optimized condition, series of studies were conducted to assess the capture performance of CTC- $\Delta$ Chip. As shown in Fig. 3b, the average capture efficiencies of BGC823 cells were around 93\% from DMEM and around $87 \%$ from healthy blood samples, respectively. Meanwhile, the recovered number showed excellent linear relationship with spiked number of cells in DMEM $\left(R^{2}=0.9994\right)$ and healthy blood samples $\left(R^{2}=0.9998\right)$. Moreover, the capture efficiencies of detecting spiked BGC823, HCT116, PC3 and SKBR3 cells into DMEM were $(93.7 \pm 3.2),(87.7 \pm 3.1),(92.3 \pm 2.5)$ and $(93.0 \pm 4.4) \%$, while the capture efficiencies of spiked BGC823, HCT116, PC3 and SKBR3 cells into blood sample were $(91.0 \pm 3.0),(86.3 \pm 3.5),(90.7 \pm 4.7)$ and $(91.3 \pm 3.1) \%$ (Fig. 3c). The fluoresce images of BGC823 were shown in Fig. 3a. Based on the "label-free" technology, CTC- $\Delta$ Chip exhibited high efficiency in isolating tumor cells in different type of cancer cell lines.

\section{Feasibility of detecting clinical samples using CTC- $\Delta$ Chip}

To validate the feasibility of detecting CTCs from clinical samples using our CTC- $\Delta$ Chip, we collected $2 \mathrm{~mL}$ peripheral blood samples from 76 cancer patients, including 20 breast cancers, 15 lung cancers, 13 esophageal cancers, 16 gastric cancers and 12 colorectal cancers. CTCs were isolated, stained and enumerated with CTC- $\Delta$ Chip according to the steps mentioned above. The detailed clinical information of them were recorded in Additional file 4, the cellular morphology and cell nucleus were shown clearly by three-color immunocytochemistry method (Additional file 5), and the enumeration results showed CTCs were detected from 53 patients $(69.7 \%)$ with the number of CTCs ranged from 0 to 30 (Additional file 6), while none of CTCs was detected in 25 healthy donors. Owing to base on the physical properties of CTCs, CTC- $\Delta$ Chip showed high performance for capturing CTCs from clinical samples.

\section{Clinical Significance of CTCs Detected by CTC- $\Delta$ Chip in GC}

To study its clinical significance of CTCs detected by our CTC- $\Delta$ Chip in GC, $2 \mathrm{~mL}$ blood samples from 40 patients (Gender: 25 males and 15 females; Mean age: 62.1 [4574] years) were collected before any treatment (Table 1). All patients included in the study were firstly diagnosed via pathological examination by endoscopic biopsy and hospitalized in Zhongnan Hospital of Wuhan University between June 1st 2017 and October 1st 2017, without a 


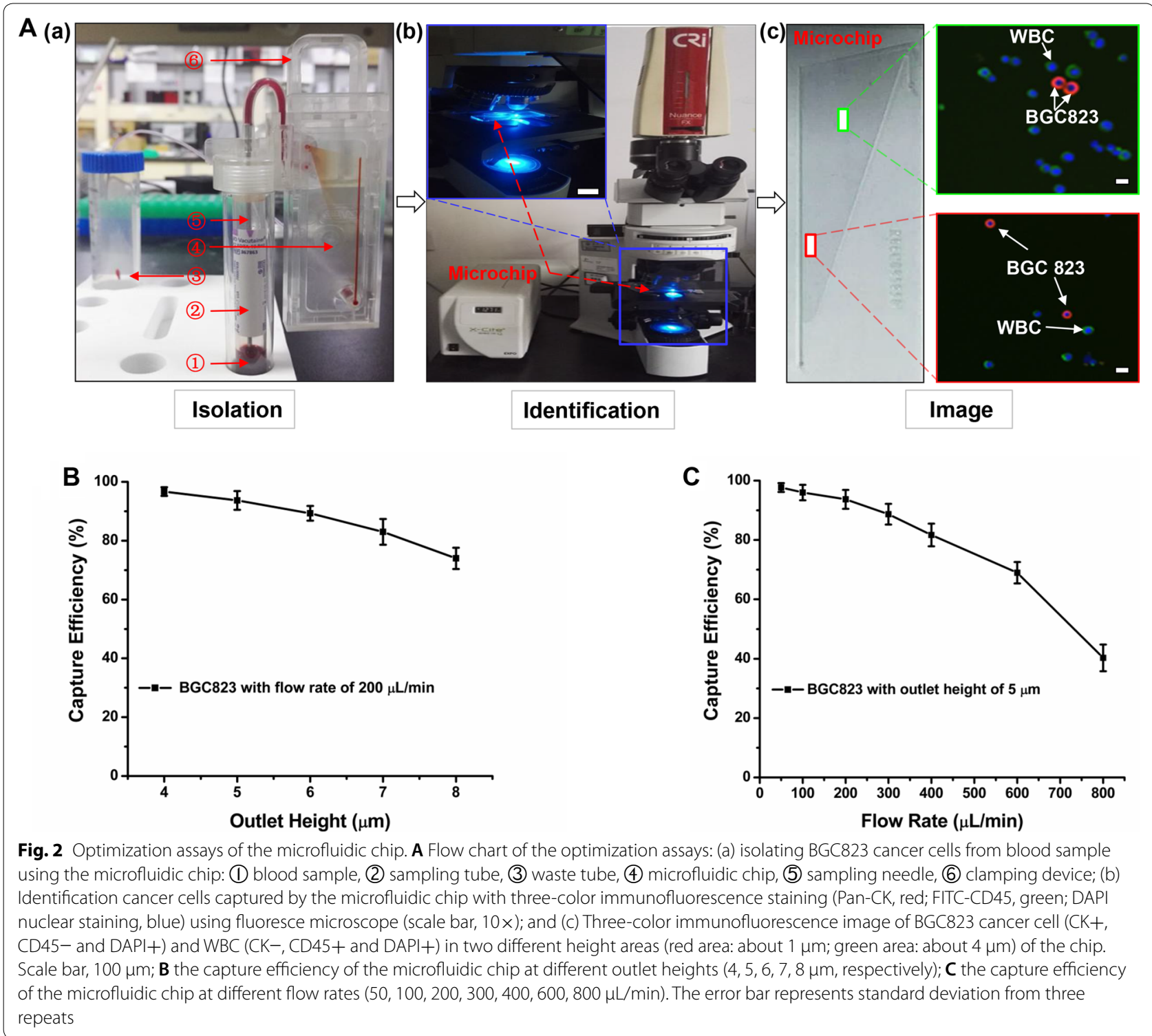

history of venous thrombosis or anticoagulation therapy, cardiovascular and cerebrovascular disease, acute or chronic inflammatory disease, blood system or immune system defects, previous malignancy.

After CTCs capture and staining in the CTC- $\Delta$ Chip, the combined information was utilized to delineate CTCs cells $(\mathrm{CK}+, \mathrm{CD} 45-$, Hoechst +$)$ from WBCs (CK-, CD45+, Hoechst+) and cellular debris (Fig. 4a). Then we summarized the counts of CTCs in Fig. $4 \mathrm{~b}$ and CTCs-positive rate in Table 2. The counts of isolated CTCs ranged from 0 to 30 per $2 \mathrm{~mL}$, with an average of $7.30 \pm 7.29$ (Mean \pm SD). Statistical analyses were used to illustrate the relationship between CTCs and clinical characteristics of GC patients. The results showed that both CTCs counts and CTCs' positive rate detected were significantly different in patients with different status of tumor differentiation $(\mathrm{P}=0.026 ; \mathrm{P}=0.030$, respectively) (Fig. 4c), lymphovascular invasion $(\mathrm{P}<0.001$; $\mathrm{P}=0.025$, respectively) (Fig. $4 \mathrm{~d}$ ), perineural invasion $(\mathrm{P}<0.001 ; \mathrm{P}=0.028$, respectively) (Fig. 4e), TNM stage $(\mathrm{P}<0.001 ; \mathrm{P}=0.033$, respectively) (Fig. 4f) and Ki-67 level $(\mathrm{P}<0.001 ; \mathrm{P}=0.024$, respectively) (Table 2). Moreover, Spearman rank correlation analysis showed that CTCs counts were highly correlated with tumor differentiation $(r=0.432, P=0.005)$, lymphovascular invasion status $(r=0.643, P<0.001)$, perineural invasion status $(\mathrm{r}=0.725, \mathrm{P}<0.001)$, Ki-67 level $(\mathrm{r}=0.677, \mathrm{P}<0.001)$ and TNM stage $(\mathrm{r}=0.789, \mathrm{P}<0.001) \quad($ Table 2$)$. However, 

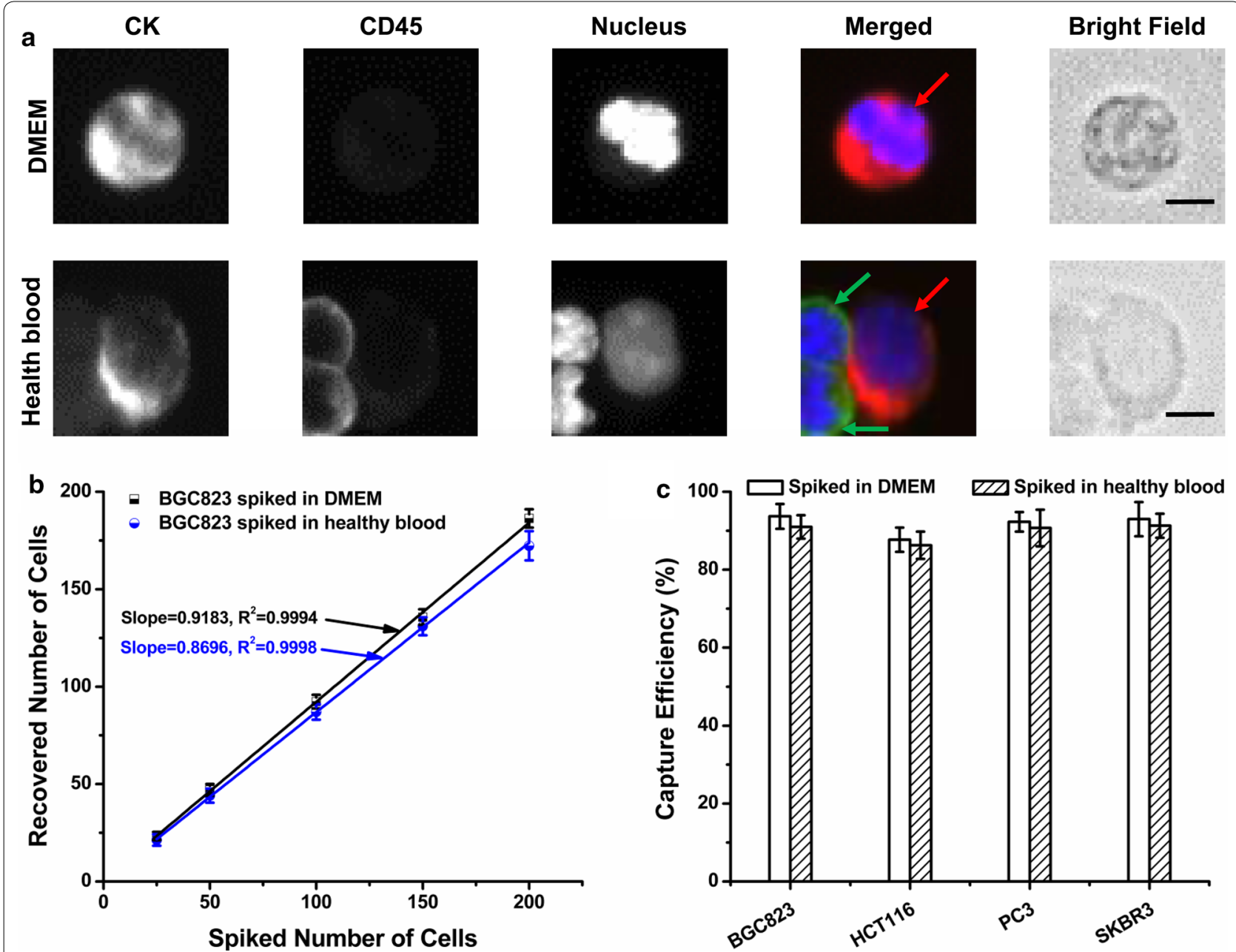

Fig. 3 Capturing performance of the CTCs enrichment platform equipped with a wedge-shaped microchamber. a The fluorescent micrographs of BGC823 captured from DMEM and healthy blood sample. Three-color immunocytochemistry method based on CK, CD45, and nuclear staining was applied to identify and enumerate BGC823 (red arrow) from WBCs (green arrow). Scale bars are $10 \mu \mathrm{m} ; \mathbf{b}$ the recovered number of BGC823 was validated from DMEM and healthy blood sample with different spiking level $(25,50,100,150,200$ cells/2 mL, respectively); c the capture efficiency of four tumor cells lines (BGC823, HCT116, PC3, SKBR3) spiked in DMEM and healthy blood samples. All experiments were performed under optimal condition. The error bar represents standard deviation from three repeats

neither the positivity rate of CTCs nor the count of CTCs was significantly correlated with age, sex, tumor location, histologic subtype (Table 2). For tumor markers, there was significant difference for the level of carcinoembryonic antigen (CEA) between CTCs' positive and CTCs' negative group $(\mathrm{p}=0.002)$, nevertheless, the significant difference did not exist in the other tumor markers level, such as carbohydrate antigen-153 (CA153), carbohydrate antigen-199 (CA199) and carbohydrate antigen-724 (CA724) $(\mathrm{P}>0.05$, respectively) (Additional file 7$)$. As the result illustrated, CTC- $\Delta$ Chip had shown high-performance in capturing CTCs from clinical samples. CTCs could be detected in $30 \mathrm{GC}$ patients (75\%) from $40 \mathrm{GC}$ patients at various stages with an average of 7.30 \pm 7.29 .
Even in patients of early stage (stage I and II, 52.9\%) and well differentiated (30\%), which exhibited a high capturing efficiency. Furthermore, CTC- $\Delta$ Chip also showed the great potential for clinical application in detecting CTCs. Both the positivity rate and the counts of CTCs were positively correlated with lymph node metastasis status and TNM stage, which implied that CTCs could be used as the supplementary to evaluate the degree of disease progression and predict the prognosis of patients.

Furthermore, we investigated the relationship between CTCs count and blood systemic inflammation indexes (Table 3). Interestingly, we found that the lymphocyte count, platelet (PLT) count, the level of neutrophil to lymphocyte ratio (NLR) and platelet to 
Table 1 Gastric cancer patient characteristics $(n=40)$

\begin{tabular}{|c|c|c|c|c|c|}
\hline Group & Patients (n) & Percentage (\%) & $\begin{array}{l}\text { Count of CTCs+ } \\
\text { patients }(n)\end{array}$ & $\begin{array}{l}\text { Count of CTCs } \\
\text { (mean } \pm \text { SD) }\end{array}$ & $\begin{array}{l}\text { CTCs } \\
\text { positive } \\
\text { rate (\%) }\end{array}$ \\
\hline \multicolumn{6}{|l|}{ Sex } \\
\hline Male & 25 & 62.5 & 19 & $7.38 \pm 7.58$ & 76.0 \\
\hline Female & 15 & 37.5 & 11 & $7.21 \pm 7.17$ & 73.3 \\
\hline \multicolumn{6}{|l|}{ Age (years) } \\
\hline$\geq 60$ & 22 & 55.0 & 17 & $6.65 \pm 6.66$ & 77.27 \\
\hline$<60$ & 18 & 45.0 & 13 & $8.18 \pm 8.20$ & 72.22 \\
\hline \multicolumn{6}{|l|}{ Tumor location } \\
\hline Cardia & 12 & 30.0 & 9 & $7.50 \pm 7.65$ & 75.0 \\
\hline Body & 14 & 35.0 & 10 & $5.71 \pm 5.44$ & 71.4 \\
\hline Pylorus & 14 & 35.0 & 11 & $8.71 \pm 8.69$ & 78.6 \\
\hline \multicolumn{6}{|l|}{ Histologic subtype } \\
\hline Adenocarcinoma & 17 & 42.5 & 13 & $5.71 \pm 5.16$ & 76.5 \\
\hline Mucinous adenocarcinoma & 11 & 27.5 & 8 & $7.73 \pm 6.72$ & 72.7 \\
\hline Signet-ring cell carcinoma & 12 & 30.0 & 9 & $9.17 \pm 10.04$ & 75.0 \\
\hline \multicolumn{6}{|l|}{ Differentiation } \\
\hline Well & 12 & 25.0 & 7 & $3.17 \pm 3.61$ & 58.3 \\
\hline Moderate & 12 & 22.5 & 9 & $6.42 \pm 5.55$ & 75.0 \\
\hline Poor & 16 & 52.5 & 14 & $11.06 \pm 8.76$ & 87.5 \\
\hline \multicolumn{6}{|l|}{ Lymphovascular invasion } \\
\hline Without & 18 & 45.0 & 10 & $2.17 \pm 2.38$ & 55.6 \\
\hline With & 22 & 55.0 & 20 & $11.50 \pm 7.29$ & 90.9 \\
\hline \multicolumn{6}{|l|}{ Perineural invasion } \\
\hline Without & 19 & 47.5 & 11 & $2.84 \pm 3.44$ & 57.9 \\
\hline With & 21 & 52.5 & 19 & $11.33 \pm 7.56$ & 90.5 \\
\hline \multicolumn{6}{|l|}{ TNM stage $^{a}$} \\
\hline Stage I & 5 & 12.5 & 2 & $0.80 \pm 1.30$ & 40.0 \\
\hline Stage II & 12 & 30.0 & 7 & $2.67 \pm 2.64$ & 58.3 \\
\hline Stage III & 16 & 40.0 & 14 & $7.88 \pm 4.33$ & 87.5 \\
\hline Stage IV & 7 & 17.5 & 7 & $18.57 \pm 7.59$ & 100.0 \\
\hline \multicolumn{6}{|l|}{ Ki-67 } \\
\hline$\geq 50 \%$ & 25 & 62.5 & 22 & $10.60 \pm 7.34$ & 88.0 \\
\hline$<50 \%$ & 15 & 37.5 & 8 & $1.80 \pm 2.01$ & 53.3 \\
\hline Total & 40 & 100.0 & 30 & $7.30 \pm 7.29$ & 75.0 \\
\hline
\end{tabular}

CTCS circulating tumor cells

a 7 th edition of AJCC/UICC classification system

lymphocyte ratio (PLR) were statistically significant between CTCs-positive and CTCs-negative group $(\mathrm{P}<0.001$, respectively; Fig. $5 \mathrm{a}-\mathrm{d})$, further Spearman rank correlation analysis showed that there was a positive correlation between the CTCs count and PLT count, NLR and PLR $(r=0.559, \mathrm{P}<0.001 ; \mathrm{r}=0.751$, $\mathrm{P}<0.001 ; \mathrm{r}=0.865, \mathrm{P}<0.001$, respectively; Fig. $5 \mathrm{e}-\mathrm{h}$ ). However, there was no significant difference between CTCs positive and negative group for leukocyte, neutrophil or monocyte $(\mathrm{P}=0.766 ; \mathrm{P}=0.691 ; \mathrm{P}=0.587$, respectively; Table 3).

\section{Discussion}

In our study, we developed a novel wedge-shaped microfluidic chip (CTC- $\Delta$ Chip) for CTCs isolation, which achieved to high efficiently isolate CTCs from less volume of patients' blood without clogging issue. In the structural of CTC- $\Delta$ Chip, the pillar-array fluid distributor made the velocity distribution of blood so linear in the entire wedge-shaped microchannel that the microchannel is not clogged by blood cells and the design of wedge-shaped microchannel provided a wide outlet also could solve the problem of outlet clogging. On this basis, 

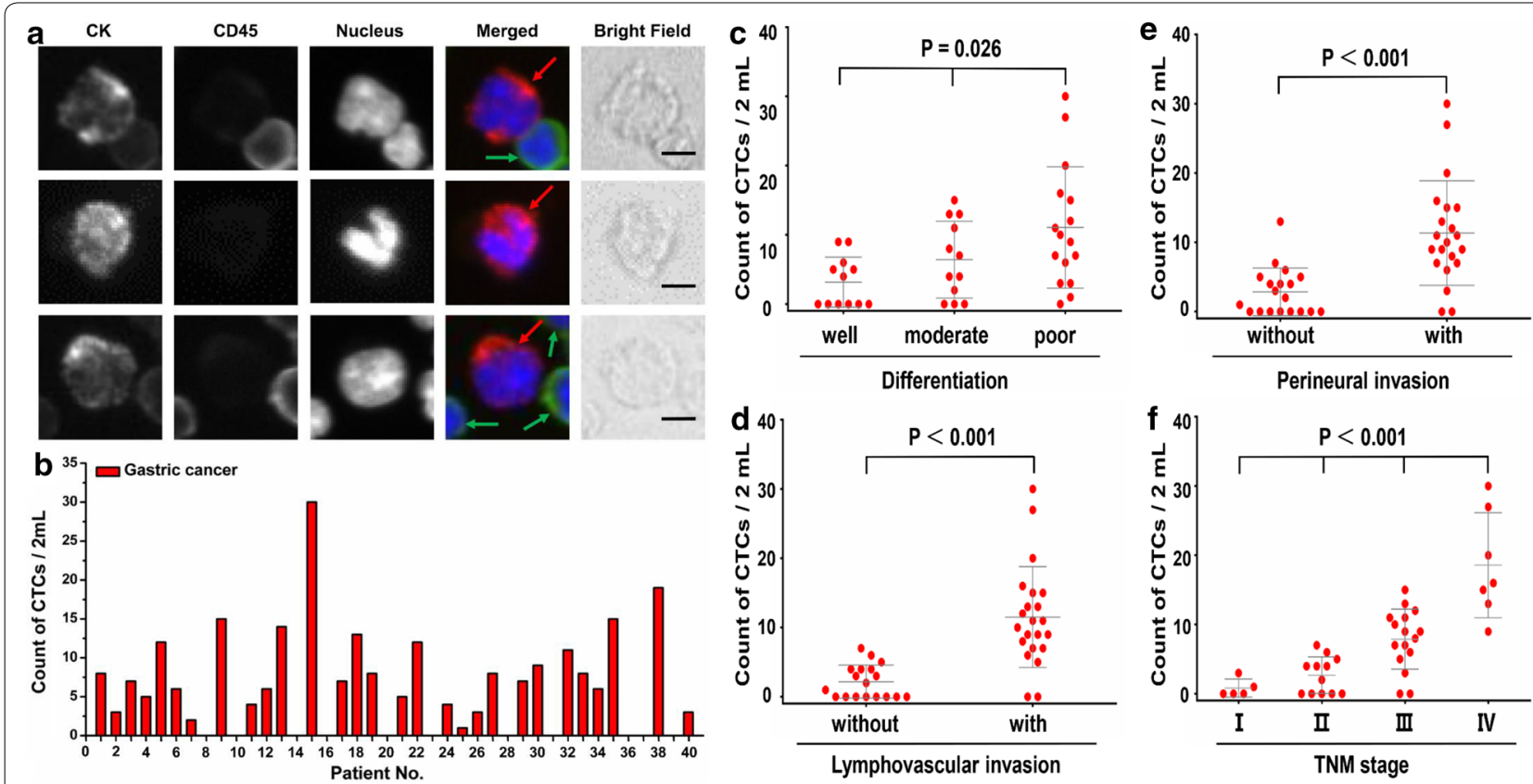

Fig. 4 Result of CTCs isolation from 40 GC patients. a CTCs detected from a GC patient. Three-color immunocytochemistry method based on CK, CD45, and nuclear staining was applied to identify and enumerate CTCs (red arrow) from non-specifically trapped WBCs (green arrow). Scale bars are $10 \mu \mathrm{m} ; \mathbf{b}$ CTCs enumeration results obtained from $40 \mathrm{GC}$ patients. Scatter plot for CTCs number of GC patients; c different tumor differential status; $\mathbf{d}$ with and without lymphovascular invasion; e with and without perineural invasion; $\mathbf{f}$ different TNM stage, each red dot stands for one GC patient. The error bars represent standard error of the mean

Table 2 The relationship of CTCs and clinicopathological variables of gastric cancer patients

\begin{tabular}{|c|c|c|c|}
\hline \multirow[t]{2}{*}{ Variable } & \multirow{2}{*}{$\begin{array}{l}\text { CTCs } \\
\text { positive } \\
\text { rate } \\
\mathrm{P}^{\mathrm{a}}\end{array}$} & \multicolumn{2}{|c|}{ Count of CTCs } \\
\hline & & $\mathrm{P}^{\mathbf{b}}$ & $P^{c}$ \\
\hline Sex & 1.000 & 0.913 & $0.915, r=0.017$ \\
\hline Age & 0.731 & 0.553 & $0.560, r=0.095$ \\
\hline Tumor location & 0.823 & 0.702 & $0.684, r=0.684$ \\
\hline Histologic subtype & 0.975 & 0.748 & $0.511, r=0.107$ \\
\hline Differentiation & 0.030 & 0.026 & $0.005, r=0.432$ \\
\hline Lymphovascular invasion & 0.025 & $<0.001$ & $<0.001, r=0.643$ \\
\hline Perineural invasion & 0.028 & $<0.001$ & $<0.001, r=0.725$ \\
\hline TNM stage $^{d}$ & 0.033 & $<0.001$ & $<0.001, r=0.789$ \\
\hline Ki-67 & 0.024 & $<0.001$ & $<0.001, r=0.677$ \\
\hline
\end{tabular}

CTCS circulating tumor cells

a $P$ value from Fisher's exact test

b $P$ value from the Mann-Whitney $U$ test

c P value from Spearman rank correlation analysis

d 7 th edition of AJCC/UICC classification system

benefiting to the biophysical properties of CTCs, such as stiffer, larger sizes, larger nuclear/cytoplasmic ratios, deformability and non-spherical bioparticles characteristic compared with other blood cells, red blood cell (RBC) would flow and randomly rotate through the microchamber, when the height of the microchamber was larger than the size of RBC. When the height of the microchamber was smaller than the diameter of RBC, RBC would be laid down due to their disk-like shape in the process of sample processing. And, due to the cut-off size of the microchamber was larger than the thickness of RBC, RBC would smoothly go through the microchamber without clogging the microfluidic chip [28, 29]. Additionally, almost $99.9 \%$ WBCs would go through CTC- $\Delta$ Chip instead of CTCs, even the cut-off size of the CTC- $\Delta$ Chip was smaller than the diameter of white blood cell (WBC). What's more, the rest subgroup of WBC left in the microchamber would be separated from CTCs with huge space resolution due to utilizing size-space amplification by the wedge-shaped microchamber. In theory, the microchamber would amplify $1 \mu \mathrm{m}$ size differential to $1 \mathrm{~mm}$ distance differential.

Importantly, CTC- $\Delta$ Chip was just fabricated by two common glass slides through wet etching technique and thermal bonding technique. Owing to the cheap raw materials and simple production processes, our CTC- $\Delta$ Chip showed the advantages of low-cost and mass-producible, and had more potential for clinical translation. Moreover, compared with the previous microfluidic devices, our chip had incorporated with 
Table 3 Association between CTCs and blood microenvironmental indexes

\begin{tabular}{|c|c|c|c|}
\hline Parameter & $C T C s+(n=30)$ & CTCs $-(n=10)$ & P value \\
\hline \multicolumn{4}{|l|}{ Immune cell } \\
\hline Leukocyte count $\left(10^{9} / \mathrm{L}\right)$ & $7.28 \pm 0.06^{\mathrm{a}}$ & $7.26 \pm 0.06$ & $0.766^{b}$ \\
\hline Neutrophil count $\left(10^{9} / \mathrm{L}\right)$ & $5.24 \pm 0.03$ & $5.24 \pm 0.02$ & $0.691^{b}$ \\
\hline Lymphocyte count $\left(10^{9} / \mathrm{L}\right)$ & $1.01 \pm 0.02$ & $1.57 \pm 0.02$ & $<0.001^{c}, r=-0.745$ \\
\hline Monocyte count $\left(10^{9} / \mathrm{L}\right)$ & $0.40 \pm 0.01$ & $0.39 \pm 0.01$ & $0.587^{b}$ \\
\hline \multicolumn{4}{|l|}{ Systemic inflammatory factor } \\
\hline $\operatorname{PLT}\left(10^{9} / \mathrm{L}\right)$ & $237.92 \pm 8.12$ & $190.14 \pm 6.17$ & $<0.001^{c}, r=0.559$ \\
\hline NLR & $5.20 \pm 0.08$ & $3.36 \pm 0.05$ & $<0.001^{c}, r=0.751$ \\
\hline PLR & $236.69 \pm 9.26$ & $125.97 \pm 3.94$ & $<0.001^{c}, r=0.865$ \\
\hline \multicolumn{4}{|l|}{ CTCS circulating tumor cells } \\
\hline \multicolumn{4}{|c|}{ a Mean \pm SEM } \\
\hline \multicolumn{4}{|c|}{ b P values from the Mann-Whitney test } \\
\hline ' $P$ values from Spearman rank & & & \\
\hline
\end{tabular}

automated high-resolution fluorescence imaging into workflow, which achieved of detecting, identifying and enumeration for CTCs efficiently and automatically, shortened the time of processing samples and reduces the error of manual operation.

Additionally, in this study, our CTC- $\triangle$ Chip also show preliminary clinical value in GC. The degree of tumor differentiation, as one of prognostic indicator for GC patients, was positively correlated with both the positivity rate and the counts of CTCs, this correlation was in correspondence with the fact that poor differentiation of tumor had the greater risk of metastasis. The correlation of lymphovascular invasion and CTCs was another part of the results needed to be discussed. Lymphovascular invasion had been proved to be an important prognostic factor of GC [30, 31]. However, in our study, data showed that both the positivity rate and CTCs count in patients with lymphovascular invasion were higher than those in patients without lymphovascular invasion, this correlation implied that lymphovascular invasion may also be a source of systemic spread of CTCs, and it might contribute to distant metastasis. Perineural invasion, as a risk factor and another prognostic factor of GC patients [32, 33], was positively correlated with both the positivity rate and the count of CTCs in our study, which implied that CTCs might be served as another high-risk factor of GC to guide clinical practice.

Noteworthy, to our knowledge, our study firstly demonstrated the relationship of CTCs and blood microenvironment indexes of GC patients under the precondition of strictly setting the inclusion criteria. Theoretically, CTCs fall off from the primary lesion into the blood circulation every day [34], most of which were eliminated in the process of interacting with the blood microenvironment and only a fairly few survived in PB. Immune cells including lymphocytes, monocytes/macrophages and neutrophils, as one of component of the blood microenvironment, played an important role during this process as the previous studies reported [35, 36]. Therefore, combining lymphocytes and CTCs might be an indicator to estimate immune condition, guide immunotherapy and predict prognosis for cancer patients. In recent years, clear evidences showed that the systemic inflammation reaction and the interaction between various inflammatory cells and the extra-cellular matrix played a crucial role in the tumor microenvironment of tumorigenesis, progression and metastasis [37-39]. Laboratory parameters that could reflect the status of systemic inflammation, had been investigated as systemic inflammatory markers, such as PLR, NLR and PLT counts [40, 41]. Previous evidences suggested that PLT might participate in the inflammatory reaction by facilitating neutrophils adhesion to endothelium through releasing chemokines and cytokines [42] and promoting tumor progression through facilitation of neoangiogenesis, production of adhesion molecules and increase of early metastatic niches [43]. The lymphocytes response was a major factor in the suppression of cancer progression [44]. The mechanisms underlying neutrophil response in tumor growth and metastasis included releasing of reactive oxygen species or nitric oxide and remodeling of the extracellular matrix [45]. Moreover, a series of metaanalysis had proved that all of them were associated with the prognosis of GC [46-48]. Based on these results, we speculated that systemic inflammatory reaction might affect the progression and metastasis of GC by the activities of CTCs, however, the specific mechanisms needed further study to clarify. 

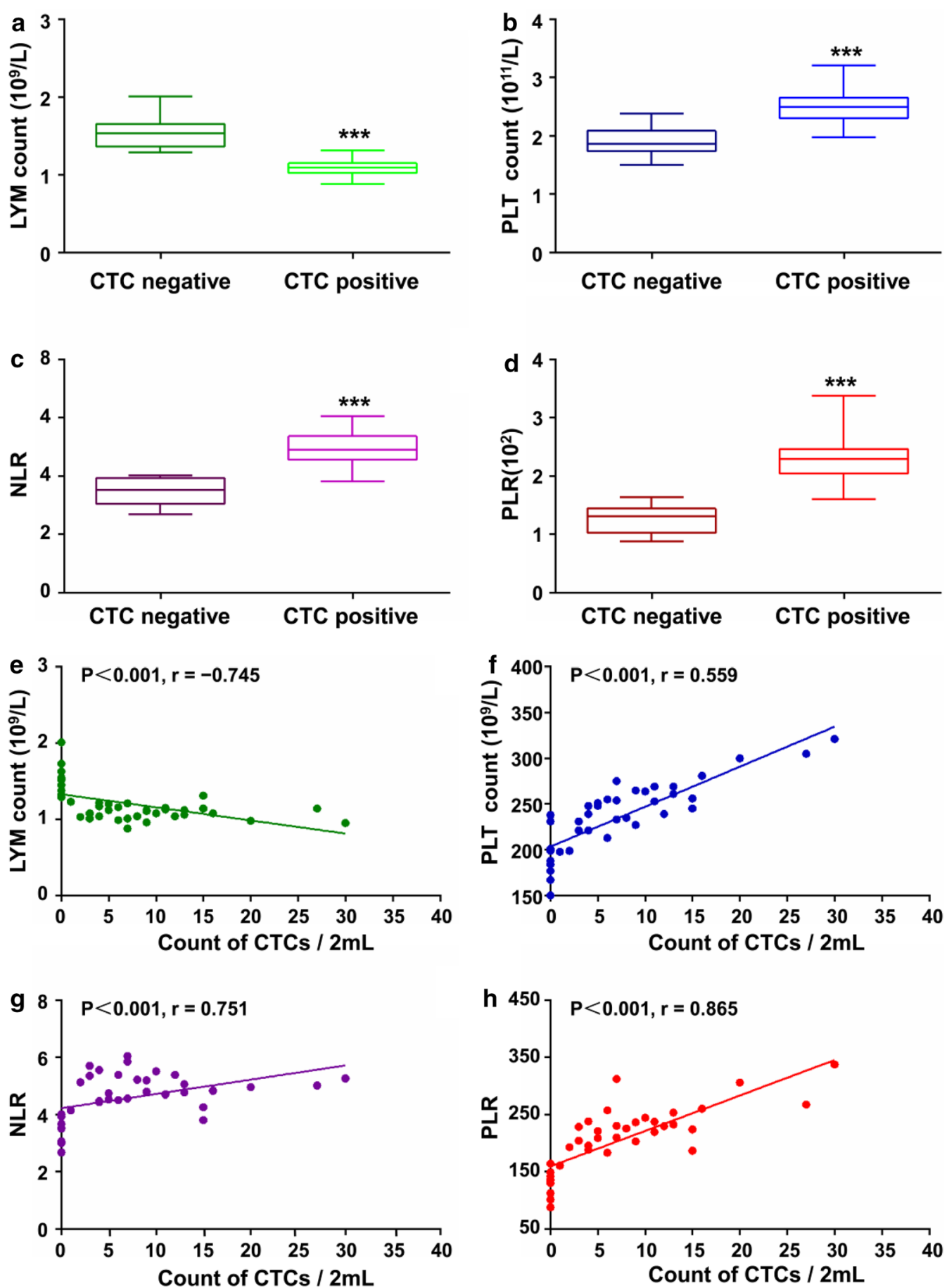

Fig. 5 Association of CTCs and blood microenvironment indexes. The difference of $\mathbf{a} L Y M$ count, $\mathbf{b}$ PLT count, $\mathbf{c}$ NLR and $\mathbf{d}$ PLR between CTCs-positive and negative group. ${ }^{* * *}$ Represents $P<0.001$. The relationship of CTC count and $\mathbf{e} L Y M$ count, $\mathbf{f}$ PLT count, $\mathbf{g}$ NLR and $\mathbf{h}$ PLR

\section{Conclusions}

In summary, we had successfully developed a low-cost CTC- $\Delta$ Chip for isolation and identification of CTCs with high-performance. Our results did not only suggest that CTC- $\Delta$ Chip had sufficient specificity and sensitivity, but also exhibited a robust platform that could accept patient's blood store for $48 \mathrm{~h}$ in $4{ }^{\circ} \mathrm{C}$ without clogging issues. More importantly, our CTC- $\Delta$ Chip had showed 
important clinical value in GC patients. Taken together, through CTCs' enumeration, CTC- $\Delta$ Chip hold great potential of clinically translating to monitoring cancer prognosis and guiding individualized treatment in the future.

\section{Additional files}

Additional file 1. The detail process of preparation of CTC- $\triangle$ Chip.

Additional file 2: Figure S1. Detailed structure of the microfluidic device.

Additional file 3. Integrated process of CTCs isolation using the CTC- $\triangle$ Chip.

Additional file 4: Table S1. Detailed clinical information and detected CTCS counts of 76 cancer patients.

Additional file 5: Figure S2. CTCs images of various type of cancers.

Additional file 6: Figure S3. Number of captured CTCs from blood samples of various type of cancer patients.

Additional file 7: Table S2. Association between CTCs and tumor markers.

\section{Abbreviations}

CTCs: circulating tumor cells; GC: gastric cancer; PLT: platelet; NLR: neutrophi to lymphocyte ratio; PLR: platelet to lymphocyte ratio; EpCAM: epithelial cell adhesion molecule; EMT: epithelial-to-mesenchymal transition; ISET: isolation by size of epithelial tumor cells; DMEM: Dulbecco's modified eagle medium; IRB: Institutional Review Board; PFA: paraformaldehyde; TNM: tumor-nodemetastasis; CEA: carcinoembryonic antigen; CA153: carbohydrate antigen-153; CA199: carbohydrate antigen-199; CA724: carbohydrate antigen-724; RBC: red blood cell; WBC: white blood cell; CK: cytokeratin.

\section{Authors' contributions}

$\mathrm{CY}$ and $\mathrm{NZ}$ contributed equally to this work. CY, NZ, KL and BX were responsible for conception and design of the study. CY, NZ and SW did the experiments, data collection, statistical analyses and writing of the manuscript. DS and CZ participated in data collection and provided statistical expertise. The report was drafted, revised, and approved by all investigators. All authors read and approved the final manuscript

\section{Author details}

1 Department of Gastrointestinal Surgery \& Department of Gastric and Colorectal Surgical Oncology, Zhongnan Hospital of Wuhan University, No.169 Donghu Road, Wuchang District, Wuhan 430071, China. ${ }^{2}$ Hubei Key Laboratory of Tumor Biological Behaviors, No.169 Donghu Road, Wuchang District, Wuhan 430071, China. ${ }^{3}$ Hubei Cancer Clinical Study Center, No.169 Donghu Road, Wuchang District, Wuhan 430071, China. ${ }^{4}$ College of Electronic and Electrical Engineering, Wuhan Textile University, No.1 Sunshine Avenue, Hongshan District, Wuhan 430200, China. ${ }^{5}$ School of Life Science and Technology, University of Electronic Science and Technology of China, No.4, Section 2, North Jianshe Road, Chengdu 610054, China.

\section{Acknowledgements}

We appreciate Dr. Pengfei Zhou and Dr. Shaoyi Huang from Wuhan YZY Medical Science and Technology Co., Ltd. for providing guidance and automatic staining \& imaging equipment.

\section{Competing interests}

The authors declare that they have no competing interests.

\section{Availability of data and materials}

The datasets supporting the conclusions of this article are included within the article.

\section{Consent for publication}

Not applicable.
Ethics approval and consent to participate

This study was approved by the Institutional Ethics Review Board of Zhongnan Hospital of Wuhan University, Wuhan, China.

\section{Funding}

This work was supported in part by following foundations: (1) National Natural Science Foundation of China (81372358, 81527801); (2) Natural Science Foundation of Hubei Province, China (2014CFA029); (3) Colleges of Hubei Province Outstanding Youth Science and Technology Innovation Team (T201305). Above funding body was not involved in the design of the study and collection, analysis, and interpretation of data and in writing the manuscript.

\section{Publisher's Note}

Springer Nature remains neutral with regard to jurisdictional claims in published maps and institutional affiliations.

Received: 6 January 2018 Accepted: 16 May 2018

Published online: 23 May 2018

\section{References}

1. Cristofanilli M, Budd GT, Ellis MJ, Stopeck A, Matera J, Miller MC, Reuben JM, Doyle GV, Allard WJ, Terstappen LW, et al. Circulating tumor cells, disease progression, and survival in metastatic breast cancer. N Engl J Med. 2004:351(8):781-91.

2. de Bono JS, Scher HI, Montgomery RB, Parker C, Miller MC, Tissing H, Doyle GV, Terstappen LW, Pienta KJ, Raghavan D. Circulating tumor cells predict survival benefit from treatment in metastatic castrationresistant prostate cancer. Clin Cancer Res Off J Am Assoc Cancer Res. 2008;14(19):6302-9

3. Okabe H, Tsunoda S, Hosogi H, Hisamori S, Tanaka E, Tanaka S, Sakai Y. Circulating tumor cells as an independent predictor of survival in advanced gastric cancer. Ann Surg Oncol. 2015;22(12):3954-61.

4. Cohen SJ, Punt CJ, lannotti N, Saidman BH, Sabbath KD, Gabrail NY, Picus J, Morse MA, Mitchell E, Miller MC, et al. Prognostic significance of circulating tumor cells in patients with metastatic colorectal cancer. Ann Oncol Off J Eur Soc Med Oncol. 2009;20(7):1223-9.

5. Normanno N, Rossi A, Morabito A, Signoriello S, Bevilacqua S, Di Maio M, Costanzo R, De Luca A, Montanino A, Gridelli C, et al. Prognostic value of circulating tumor cells' reduction in patients with extensive small-cell lung cancer. Lung Cancer. 2014:85(2):314-9.

6. Racila E, Euhus D, Weiss AJ, Rao C, McConnell J, Terstappen LW, Uhr JW. Detection and characterization of carcinoma cells in the blood. Proc Natl Acad Sci USA. 1998;95(8):4589-94.

7. Zieglschmid V, Hollmann C, Bocher O. Detection of disseminated tumor cells in peripheral blood. Crit Rev Clin Lab Sci. 2005;42(2):155-96.

8. Weissenstein U, Schumann A, Reif M, Link S, Toffol-Schmidt UD, Heusser P. Detection of circulating tumor cells in blood of metastatic breast cancer patients using a combination of cytokeratin and EpCAM antibodies. BMC Cancer. 2012:12:206.

9. Liu Z, Zhang W, Huang F, Feng H, Shu W, Xu X, Chen Y. High throughput capture of circulating tumor cells using an integrated microfluidic system. Biosens Bioelectron. 2013;47:113-9.

10. Huang T, Jia CP, Jun Y, Sun WJ, Wang WT, Zhang HL, Cong H, Jing FX, Mao $\mathrm{HJ}$, Jin QH, et al. Highly sensitive enumeration of circulating tumor cells in lung cancer patients using a size-based filtration microfluidic chip. Biosens Bioelectron. 2014;51:213-8.

11. Fan X, Jia C, Yang J, Li G, Mao H, Jin Q, Zhao J. A microfluidic chip integrated with a high-density PDMS-based microfiltration membrane for rapid isolation and detection of circulating tumor cells. Biosens Bioelectron. 2015:71:380-6.

12. Nwankire CE, Venkatanarayanan A, Glennon T, Keyes TE, Forster RJ, Ducree J. Label-free impedance detection of cancer cells from whole blood on an integrated centrifugal microfluidic platform. Biosens Bioelectron. 2015:68:382-9.

13. Shen H, Yang J, Chen Z, Chen X, Wang L, Hu J, Ji F, Xie G, Feng W. A novel label-free and reusable electrochemical cytosensor for highly sensitive detection and specific collection of CTCs. Biosens Bioelectron. 2016:81:495-502. 
14. Kwak B, Lee J, Lee D, Lee K, Kwon O, Kang S, Kim Y. Selective isolation of magnetic nanoparticle-mediated heterogeneity subpopulation of circulating tumor cells using magnetic gradient based microfluidic system. Biosens Bioelectron. 2017;88:153-8.

15. Warkiani ME, Khoo BL, Wu L, Tay AK, Bhagat AA, Han J, Lim CT. Ultra-fast, label-free isolation of circulating tumor cells from blood using spiral microfluidics. Nat Protoc. 2016;11(1):134-48.

16. Saliba AE, Saias L, Psychari E, Minc N, Simon D, Bidard FC, Mathiot C, Pierga JY, Fraisier V, Salamero J, et al. Microfluidic sorting and multimodal typing of cancer cells in self-assembled magnetic arrays. Proc Natl Acad Sci USA. 2010;107(33):14524-9.

17. Jo SM, Lee JJ, Heu W, Kim HS. Nanotentacle-structured magnetic particles for efficient capture of circulating tumor cells. Small. 2015;11 (16):1975-82.

18. Liljefors M, Nilsson B, Fagerberg J, Ragnhammar P, Mellstedt H, Frodin JE. Clinical effects of a chimeric anti-EpCAM monoclonal antibody in combination with granulocyte-macrophage colony-stimulating factor in patients with metastatic colorectal carcinoma. Int J Oncol. 2005;26(6):1581-9.

19. Gorges TM, Tinhofer I, Drosch M, Rose L, Zollner TM, Krahn T, von Ahsen O. Circulating tumour cells escape from EpCAM-based detection due to epithelial-to-mesenchymal transition. BMC Cancer. 2012;12:178.

20. Hosokawa M, Hayata T, Fukuda Y, Arakaki A, Yoshino T, Tanaka T, Matsunaga T. Size-selective microcavity array for rapid and efficient detection of circulating tumor cells. Anal Chem. 2010;82(15):6629-35.

21. Zheng S, Lin H, Liu JQ, Balic M, Datar R, Cote RJ, Tai YC. Membrane microfilter device for selective capture, electrolysis and genomic analysis of human circulating tumor cells. J Chromatogr A. 2007:1162(2):154-61.

22. Chen F, Wang S, Fang Y, Zheng L, Zhi X, Cheng B, Chen Y, Zhang C, Shi D, Song $\mathrm{H}$, et al. Feasibility of a novel one-stop ISET device to capture CTCS and its clinical application. Oncotarget. 2017;8(2):3029-41.

23. Li Y, Gong J, Zhang Q, Lu Z, Gao J, Li Y, Cao Y, Shen L. Dynamic monitoring of circulating tumour cells to evaluate therapeutic efficacy in advanced gastric cancer. Br J Cancer. 2016;114(2):138-45.

24. Zhou J, Ma X, Bi F, Liu M. Clinical significance of circulating tumor cells in gastric cancer patients. Oncotarget. 2017;8(15):25713-20

25. Matsusaka S, Chin K, Ogura M, Suenaga M, Shinozaki E, Mishima Y, Terui $Y$, Mizunuma N, Hatake K. Circulating tumor cells as a surrogate marker for determining response to chemotherapy in patients with advanced gastric cancer. Cancer Sci. 2010;101(4):1067-71.

26. Dhar M, Wong J, Karimi A, Che J, Renier C, Matsumoto M, Triboulet M, Garon EB, Goldman JW, Rettig MB, et al. High efficiency vortex trapping of circulating tumor cells. Biomicrofluidics. 2015:9(6):064116.

27. Sobin LH, Gospodarowicz MK, Wittekind C. TNM Classification of Malignant Tumours. 7th ed. Oxford: Blackwell Publishing Ltd; 2010.

28. Hsieh TH, Keh HJ. Boundary effects on electrophoresis of a colloidal cylinder with a nonuniform zeta potential distribution. J Colloid Interface Sci. 2007;315(1):343-54.

29. Davison SM, Sharp KV. Transient simulations of the electrophoretic motion of a cylindrical particle through a $90^{\circ}$ corner. Microfluid Nanofluid. 2008;4(5):409-18.

30. Zhao LY, Chen XL, Wang YG, Xin Y, Zhang WH, Wang YS, Chen XZ, Yang $K$, Liu K, Xue L, et al. A new predictive model combined of tumor size, lymph nodes count and lymphovascular invasion for survival prognosis in patients with lymph node-negative gastric cancer. Oncotarget. 2016;7(44):72300-10.

31. Lee JH, Kim MG, Jung MS, Kwon SJ. Prognostic significance of lymphovascular invasion in node-negative gastric cancer. World J Surg. 2015;39(3):732-9.
32. Aurello P, Berardi G, Tierno SM, Rampioni Vinciguerra GL, Socciarelli F, Laracca GG, Giulitti D, Pilozzi E, Ramacciato G. Influence of perineural invasion in predicting overall survival and disease-free survival in patients with locally advanced gastric cancer. Am J Surg. 2017;213(4):748-53. https://doi.org/10.1016/j.amjsurg.2016.05.022.

33. Selcukbiricik F, Tural D, Buyukunal E, Serdengecti S. Perineural invasion independent prognostic factors in patients with gastric cancer undergoing curative resection. Asian Pac J Cancer Prev APJCP. 2012;13(7):3149-52.

34. Chang YS, di Tomaso E, McDonald DM, Jones R, Jain RK, Munn LL. Mosaic blood vessels in tumors: frequency of cancer cells in contact with flowing blood. Proc Natl Acad Sci USA. 2000;97(26):14608-13.

35. De Giorgi U, Valero V, Rohren E, Mego M, Doyle GV, Miller MC, Ueno NT, Handy BC, Reuben JM, Macapinlac HA, et al. Circulating tumor cells and bone metastases as detected by FDG-PET/CT in patients with metastatic breast cancer. Ann Oncol Off J Eur Soc Med Oncol. 2010;21(1):33-9.

36. De Giorgi U, Mego M, Scarpi E, Giuliano M, Giordano A, Reuben JM, Valero V, Ueno NT, Hortobagyi GN, Cristofanilli M. Relationship between lymphocytopenia and circulating tumor cells as prognostic factors for overall survival in metastatic breast cancer. Clin Breast Cancer. 2012;12(4):264-9.

37. Mantovani A, Allavena P, Sica A, Balkwill F. Cancer-related inflammation. Nature. 2008:454(7203):436-44.

38. Colotta F, Allavena P, Sica A, Garlanda C, Mantovani A. Cancer-related inflammation, the seventh hallmark of cancer: links to genetic instability. Carcinogenesis. 2009;30(7):1073-81.

39. Grivennikov SI, Greten FR, Karin M. Immunity, inflammation, and cancer. Cell. 2010;140(6):883-99.

40. Xu AM, Huang L, Zhu L, Wei ZJ. Significance of peripheral neutrophillymphocyte ratio among gastric cancer patients and construction of a treatment-predictive model: a study based on 1131 cases. Am J Cancer Res. 2014;4(2):189-95.

41. Kim EY, Lee JW, Yoo HM, Park CH, Song KY. The platelet-to-lymphocyte ratio versus neutrophil-to-lymphocyte ratio: which is better as a prognostic factor in gastric cancer? Ann Surg Oncol. 2015;22(13):4363-70.

42. Jenne CN, Urrutia R, Kubes P. Platelets: bridging hemostasis, inflammation, and immunity. Int J Lab Hematol. 2013;35(3):254-61.

43. Labelle M, Begum S, Hynes RO. Platelets guide the formation of early metastatic niches. Proc Natl Acad Sci USA. 2014;111(30):E3053-61.

44. Dunn GP, Old LJ, Schreiber RD. The immunobiology of cancer immunosurveillance and immunoediting. Immunity. 2004;21 (2):137-48.

45. De Larco JE, Wuertz BR, Furcht LT. The potential role of neutrophils in promoting the metastatic phenotype of tumors releasing interleukin-8. Clin Cancer Res Off J Am Assoc Cancer Res. 2004:10(15):4895-900.

46. Zhang $X$, Zhang W, Feng LJ. Prognostic significance of neutrophil lymphocyte ratio in patients with gastric cancer: a meta-analysis. PLoS ONE. 2014;9(11):e111906.

47. Xin-Ji Z, Yong-Gang L, Xiao-Jun S, Xiao-Wu C, Dong Z, Da-Jian Z. The prognostic role of neutrophils to lymphocytes ratio and platelet count in gastric cancer: a meta-analysis. Int J Surg. 2015;21:84-91.

48. Xu Z, Xu W, Cheng H, Shen W, Ying J, Cheng F, Xu W. The prognostic role of the platelet-lymphocytes ratio in gastric cancer: a meta-analysis. PLoS ONE. 2016;11(9):e0163719.

Ready to submit your research? Choose BMC and benefit from:

- fast, convenient online submission

- thorough peer review by experienced researchers in your field

- rapid publication on acceptance

- support for research data, including large and complex data types

- gold Open Access which fosters wider collaboration and increased citations

- maximum visibility for your research: over 100M website views per year

At BMC, research is always in progress.

Learn more biomedcentral.com/submissions 